\title{
Origin of cosmic rays
}

\author{
V. A. Dogiel \\ P.N.Lebedev Institute of Physics, Leninskii pr, 53, 119991 Moscow, Russia, \\ email: dogiel@lpi.ru
}

\begin{abstract}
Cosmis rays are an essential component of the interstellar medium because of their high energy density. This paper reviews the origin of cosmic rays.
\end{abstract}

Keywords. cosmic rays, acceleration of particles, magnetic fields, ISM: bubbles

Cosmic rays (CRs) in spite of their small density, $\sim 10^{-10} \mathrm{~cm}^{-3}$ in comparison with the average gas density in the Galactic disk, $\sim 1 \mathrm{~cm}^{-3}$, are an essential component of the interstellar medium because their energy density is high. It is about $\sim 1 \mathrm{eV} \mathrm{cm}^{-3}$, that is comparable with that of interstellar magnetic fields and turbulent motions of gas/plasma in the Galactic disk. In this respect, analyses of interstellar plasma dynamics, ionization, heating etc. in the Galaxy is impossible without taking into consideration of CR influence.

Effect of CRs was found in 1785 by de Coulomb who observed a spontaneous discharge of electroscopes. Though the origin of this effect was unclear. The explanation of this phenomenon came only in the beginning of the 20th century and paved the way to one of mankind's revolutionary scientific discoveries. Exactly one hundred years ago Victor Hess concluded from his balloon experiments that the effect of discharge is due to an unknown radiation of extraterrestrial origin: "a radiation of very high penetrating power enters our atmosphere from above and produces .... ionization in closed vessels".

Further experiments showed that this radiation consisted of high energy particles, mainly protons, with the energies higher than above $1 \mathrm{GeV}$. But it took almost another hundred years in order to understand the origin of radiation.

The first model of CR origin was suggested by Baade and Zwicky in 1934. They assumed that CRs uniformly filled the whole volume of the Universe, and they were produced by supernovae (SNe) which exploded once per $10^{3}$ yr in each $(300 \mathrm{pc})^{3}$ volume of the Universe. This idea of extragalactic origin of CRs was criticized by Ginzburg (1972) who suggested a test to prove the galactic CR origin from $\gamma$-ray data which ought to show fluctuations of $\mathrm{CR}$ density in galaxies. His idea was confirmed from subsequent measurements on the Compton and Fermi $\gamma$-ray satellites.

The main problem for the galactic theory of CRs was to explain very specific parameter of their flux, that the spectrum is power-law over six decades, from $\sim 10^{9}$ to $3 \times 10^{15} \mathrm{eV}$ with a single spectral index, $\propto E^{-2.7}$. The first idea about CR acceleration in the Galaxy was suggested by Fermi $(1949,1954)$, who assumed that the acceleration took place in the whole volume of the Galaxy by collisions of charged particles with moving fluctuations of magnetic field (magnetic clouds). Advantages of this mechanism was that it produced CRs everywhere in the Galaxy with a power-law spectrum. However, the spectrum is too hard, $\propto E^{-1}$, in comparison with the spectrum of galactic CRs. Secondly, acceleration efficiency of this mechanism is quite low. Hundred million years is required in order to increase the energy of particles only in three times. So, further ideas about acceleration processes in the Galaxy were required.

From the analysis of CR chemical composition it was estimated that the total luminosity of $\mathrm{CR}$ in the Galaxy is about $L_{\mathrm{CR}} \sim 3 \times 10^{40} \mathrm{erg} \mathrm{s}^{-1}$. Then from energetic 
considerations it was concluded that the most probable CR sources in our Galaxy could be galactic SNe because their energy output is about $10^{42} \mathrm{erg} \mathrm{s}^{-1}$, and if only a few per cents of these energy is transformed into CR, it is enough to produce the observed CR flux (see for details Ginzburg \& Syrovatskii 1964 and Berezinskii et al. 1990). The question was how SNe being in different parts of the Galaxy generate a universal spectrum with the index close to -2. The answer was obtained in 1977 when Axford et al. (1977), Krymskii (1977) and others suggested a model of CR acceleration near shock fronts. Advantages of this model was that the efficiency of acceleration was much higher than that of the classical Fermi acceleration. The mechanism produces a universal power-law spectrum of CRs, $\propto E^{-2}$, independently of medium conditions. And just SN explosions produce shock fronts in the Galactic disk. However, the maximum energy of accelerated particles is determined by the shock lifetime and cannot exceed $E_{\max } \sim 10^{14} \mathrm{eV}$ (see e.g. Berezhko \& Völk 2000). This means that there are other sources of CR production in the Galaxy. Recent Fermi-LAT observations found indeed regions where particles can be accelerated up to energies higher than $10^{14} \mathrm{eV}$. This telescope found a cocoon of freshly accelerated cosmic rays in the Cyg OB association (see Ackermann et al. 2011) that suggests that $\mathrm{OB}$ associations and their superbubbles are likely the source of a substantial fraction of galactic cosmic rays from the collective action of multiple shocks from supernovae and the winds of massive stars where particles can be accelerated up to energies $10^{18} \mathrm{eV}$ (see Bykov \& Toptygin 2001). Recent discovery of a mysterious, diffuse giant gamma-ray structures, Fermi bubbles, (see Dobler et al. 2010 and $\mathrm{Su}$ et al. 2010) may indicate another source of CR generation in the Galaxy. These structures may result from star captures by the central black hole. Each capture produces a shock propagating into the Galactic halo where shocks accelerate electrons (up to $E \sim 1 \mathrm{TeV}$ ) and protons with energies up to $E \sim 10^{18} \mathrm{eV}$ (see Cheng et al. 2011, 2012).

Thus, though many aspects of CR origin are clear now, this theory cannot be considered complete. Future experiments may modify or even change our ideas about CR origin.

\section{Acknowledgements}

VAD is partly supported by the RFFI grant 12-02-00005-a and ISSI.

\section{References}

Ackermann, M., Ajello, M., Allafort, A., et al. 2011, Science 334, 1103

Axford,W. I., Leer, E. \& Skadron, G. 1977, Proc. 15th Int. Cosmic Ray Conf. 11, 132

Baade, W. \& Zwicky, F. 1934, Phys. Rev. 46, 76

Bell, A. R. 1978, MNRAS 182, 147

Berezhko, E. G. \& Völk, H. J. 2000, A\&A $A$ 357, 283

Berezinskii, V. S., Bulanov, S. V., Dogiel, V. A., Ginzburg, V. L., \& Ptuskin, V. S. 1990, Astrophysics of Cosmic Rays, ed. V. L. Ginzburg, Norht-Holland: Amsterdam

Blandford, R. D. \& Ostriker, J. P. 1978, ApJ (Letters) 221, L29

Bykov, A. M. \& Toptygin, I. N. 2001, Astron. Lett. 27, 625

Cheng, K.-S., Chernyshov, D. O., Dogiel, V. A., et al. 2011, ApJ (Letters) 731, L17

Cheng, K.-S., Chernyshov, D. O., Dogiel, V. A., et al. 2012, ApJ 746, 116

Dobler, G., Finkbeiner, D. P., Cholis, I., et al. 2010, ApJ 717, 825

Fermi, E. 1949, Phys. Rev. 75, 1169

Fermi, E. 1954, ApJ 119, 1

Ginzburg, V. L. 1972, Nature Phys. Sci. 239, 8

Ginzburg, V. L. \& Syrovatskii, S. I. 1964, The Origin of Cosmic Rays, New York: Macmillan

Krymskii, G. F. 1977, Soviet Physics - Doklady 22, 327

Su, M., Slatyer, T. R., \& Finkbeiner, D. P. 2010, ApJ 724, 1044 